\title{
Related Discussion on Agent-oriented Programming
}

\author{
Hou Yan
}

Qilu Normal University, Shandong, Jinan, 250014

\section{Keywords: Agent-oriented; Program design; Characteristic; Design framework}

\begin{abstract}
With the continuous development of computer technology in recent years, Agent system is considered to be a new and effective technology in the complex system development process, which is supportive in open environment. Therefore, much attention has been paid to Agent-oriented program design, and AOP has received wide attention in the field of software engineering, artificial intelligence, etc., which further improves the application efficiency of AOP. This paper discusses the related problems of Agent-oriented programming and puts forward Agent-oriented programming framework.

With the continuous development of computer technology and Internet widespread application, all kinds of application software emerge endlessly, but there are a lot of requirements with a variety of complex characteristics, such as independent, initiative to adapting, and these characteristics have created new challenges and opportunities for the current mainstream object-oriented approach. With increasingly deep Agent studies, there is greater impact on software system and the design of smart structures, and it has important use value in overcoming software crisis. With different research problems, Agent has different definitions. One of the most common is defined that Agent is designed for certain tasks, which can play a unique role in the system also has its own life cycle. Agent, as an autonomous software, in addition to its characteristics description, the intelligent analysis of software should be done to know the software's dynamic running state or type, and describe main purpose and the purpose of using Agent. In software design, Agent must have independent ability, social ability, etc., and its behavior must conform to certain rules.
\end{abstract}

\section{THE RELATED CONCEPTS OF AGENT-ORIENTED PROGRAMMING}

Agent-oriented software engineering is an emerging example in software engineering field research, but it has laid a good foundation and premise for software development. Agent software engineering in the new study field widely uses some close descriptive terms. Agent-oriented programming (AOP) is a kind of improvement and development of object-oriented program design and the program design is close to the program design realization level. Agent-oriented analysis (AOA) analyzes and develops oriented object, understands the demand and function of the Agent software development, and finally sets designated Agent. Agent-oriented programming is a kind of object-oriented software design, also is the demand and function of the development process. So Agent can accurately complete the assigned tasks by the user or hardware device.

\section{THE GENERAL SITUATION OF AGENT-ORIENTED PROGRAMMING}

Agent-oriented programming studies object-oriented software design and its main purpose is the development and maintenance of low cost and tools method based on Agent software. In the process of software design, pay attention to the flexibility and practicability. According to the changes of The Times in the future development, effectively upgrade. Agent-oriented programming is a kind of extension and expansion of object-oriented program design and program design is seen as the inheritance and development of structure programming. Agent-oriented programming mainly takes entity as the object, can integrate the logical combinations of object, and abstracts objective entity. It is seen as the inheritance and development of the object. Although the abstract can be improved to some extent, but there is a great similarity between the Agent and the object, and it plays a good supportive role in intellectual composition structure. Agent has the effect of interaction relationship between supportive Agents, and plays a very important role in the relationship between senior 
Agents. It can realize communication between Agents. The differences between Agent-oriented programming and object oriented program design are mainly reflected in that outside world is binding the objects, but the Agent cannot get outside help to achieve direct jurisdiction of behavior, so analysis method of OOA and OOD can get a promotion and application. This article mainly adopts responsibility driven design method to construct a more practical AOA and AOD methods, whose main advantage lies in: first, the system can divide angle and granularity. Object-oriented design can have many aspects of reference standard, but it is hard to master well in actual use, so many designers dislike it. Agent-oriented ideology has strong direction, and analysts in the process of analysis are able to clearly guide principles and analyze the angle and granularity. Second, it can divide the analysis and design phase. OO method analyzes and designs in the same way, but it is difficult to distinguish between two ways of tasks in the actual use. Agent-oriented method goals and objects in different stages also are very different, so they can be clearly identified in analysis and keep consistent in style and features. There are interactions between systems. Each part of the system can realize mutual interaction and work in the system as the core of intelligence. The interaction between Agents is not a simple communication conversation between Agents, but involves various factors such as Agent tasks and design and so on, so the purpose of traditional object-oriented way is therefore difficult to perform task requirements. The fourth is that the system is very smart in complex problem solving. Agent has brought a whole new kind of design point of view in the field of artificial intelligence, and solves a problem as the integral in the system solving process to achieve the rationality and coordination of allocation and solving problems. It can improve some disadvantages in traditional system design. Agent-oriented programming method has great advantages in the analysis and solving of complex systems.

\section{AGENT-ORIENTED ANALYSIS AND DESIGN METHOD}

\section{Agent-oriented programming analysis and method}

Gaia theory has certain drawbacks in the aspect of Agent-oriented programming. The theory cannot be applied to openness and unpredictable Internet, but has good development prospects in the development closed domain. Gaia method limits the results of program design, and improves Internet application development. It has a certain supporting function in Agent system in open environment. In Agent design program, Gaia method is divided into two aspects. In the micro level, Agent can realize its own architecture and organization; in the macro aspect, it frames the interaction relationship and organizational relationship between Agents; in the process of running keep Agent static. Gaia method does not exhibit its own characteristics in Agent independent behavior and problems. In terms of Agent interaction and architectural aspects, organization structure has not effectively modeling. Use Gaia method, designers need to make systematic software development and utilization. In the process of analysis, Gaia method need to find system roles, then interact Agents role for modeling, and form characters into different properties and categories. Responsibilities attributes mainly add some role contents in the system, and protect the safety of system. License properties mainly allow the system to make some behavior, and allow certain information to be accessed. Roles complete system task in the separate process. Agreement is a kind of special mode under the interaction. In order to show the attributes of the characters, Gaia method models role in the operation of these roles and forms corresponding operation rules, and establishes some illustration for design and analysis. In the process of design, Gaia method firstly draws a role in Agent class, then makes actual number, determines one or more roles of the Agent, and provides the corresponding service type. Finally Agent implements communication with Agents with corresponding model.

\section{Multi-agent software analysis and design method}

Multi-agent software engineering design method was put forward by WOOd and Deloach, and has a great deal of similarity in the application field with Gaia theory. But Agent program design mainly uses tools to code, and the main purpose is to find the prove method for the current few Agent system research and forms industrialization kit. Multi-agent program design mainly requires the designer out of traditional design mode and thinking, and achieves a variety of design methods. 
Multi-agent program design and Gaia theory in finite field has a great deal of similarity, the interaction between the Agent did not appear the corresponding, and a single pass.

Multi-agent program design method is divided into seven steps. The first step is mainly to look for targets, and then makes the transition of systems and hierarchical settings of the structure. The first step firstly recognizes the system effectively, classifies targets, and finally sets the title. The second step is to apply for use cases, establishes system initial examples and sequence diagram, introduces different roles in the system, and realizes system space interaction and organization. Sequence diagrams mainly position the system roles and relay messages between the characters. The third step is to refine characters. This stage needs to create a suitable target, and positions the role of on one of the goals. The task is to position relative roles and determines the use state of tasks. The fourth step is mainly to establish Agent category. Draw the characters in Agent category, and the diagram class is similar to the figure class of object. But related semantics is senior session and is similar with the inheritance structure in state diagram. The fifth step is to adjust the coordination protocol in state diagram and create and adjust the state of the Agent. Sixth step mainly gathers Agent class, summarizes the internal functions, and classifies according to different functions appropriately. The last step is mainly to design the system, identify Agent types and models, and describe the types of Agent in development diagram. Multi-agent program design is able to complete development diagram code's automatic generation in the future development.

\section{Database information system modeling}

Wagner put forward Agent object relations (AOR) model, which is similar in the aspect of information system. AOR is more of extensive application in database model. The main design purpose of ER meta model is to transform the relation between different information systems, which plays a supporting role in static entity conversion, but can reduce the role of entity activities in information system. AOR model development is mainly to expand ER model, and makes modeling processing of Agent relationship outside the static entities. Agent models have six different entities types to complete Agent commission and meet the requirements of the Agent. A set of sub Agent models is an organization, and the Agent subsystems are able to meet the requirements of the system and do specific task.

But in organization testing requirements and related events, make Agent be responsible, and it has a great deal of consistency with Gaia method.

\section{CONCLUSION}

In the development of complex system, Agent technology plays an important role. Agent-oriented software engineering method is one of the important design methods in software system analysis, design, and architecture process, and it provides a more comprehensive analysis for Agent software engineering method.

\section{REFERENCES}

[1] Mao Xinjun, Hu Cuiyun, Sun Yuekun. Study of Agent-oriented programming [J]. Journal of Software, 2012, (11) : 2885-2904.

[2] Mao Xinjun, Chen Yin, Zhang Tingting. Programming mode and language supporting social technology system structure [J]. Journal of National University of Defense Technology, 2014, (3) : 103-110.

[3] Zhang Ying. Agent-oriented programming research [J]. Information and Computers, 2015, (17) : 128-129.

[4] Yang Zhigang. Subject-oriented programming based on Agent-O [J]. Computer Programming Skills and Maintenance, 2014, (16) : 4-5, 8.

[5] Liu Zhen, Zhang Zhizheng. B language description action model method based on ILP and ASP [J]. Journal of Computer Science, and 2015 (1) : 220-226.

[6] Hu Cuiyun, Mao Xinjun, Chen Yin. Agent-oriented programming and language Oragent based on organization [C] The 4th China Agent theory and application academic proceedings. 2012:1-14. 